\title{
Delivering electronic prescribing and medicines administration in challenging areas such as paediatrics and maternity at King's College Hospital NHS Foundation Trust
}

\author{
Authors: Hardeep Sahota, Peter Hughes, Chris Barrass and Ben Fidler
}

\section{Aims}

In 2013, the government announced that all NHS organisations must be paperless by 2018 . The aim of my project was to design, implement and maintain six paediatric wards, which included HDU, ICU and four maternity locations, within a short 6-month time frame.

\section{Methods}

The primary process involved the electronic prescribing and medicines administration (EPMA) team understanding and mapping paper processes and deciding, along with ward staff, what paper processes worked and what needed to change. The team worked with this basic ethos in mind: if the existing process could be replicated electronically, then the process would be scoped for the electronic go-live. Our focus was clear: to get as much of the trust as paperless as quickly as possible. My job was to identify key stakeholders and engage with them from the beginning of design review and past maintenance of the system. It was important to use a multidisciplinary approach, much like any other clinical setting. My job was to facilitate the decisionmaking process by ensuring that stakeholders realised that key choices were being made by them. I felt that this was essential in ensuring that clinical staff bought into the notion that this was their system, designed to improve patient experience and safety. Strict timelines were set to ensure that all clinical build was designed, built, tested and then approved by the clinical staff.

A go-live date was agreed with all staff. A rolling approach was decided on: each ward went once a month, with 24-hour go-live support for 1 week followed by reduced 12-hour shifts by the EPMA team. A member of the EPMA team was also available over 24 hours via switchboard, for any technical query.

\section{Results}

As planned, all paediatric and maternity locations went live with EPMA within the 6 months.

Authors: King's College Hospital NHS Foundation Trust, London, UK

\section{Conclusions}

Phase two of the project was a great success. The paediatric units have commented on the clarity and ease of medication rounds. Doctors like the fact that they have commonly prescribed medications with pre-populated doses and frequencies; they have also commented on the fact that discharge documentation is electronically sent to general practice, which really highlights the benefit with transfer of care between primary and secondary care. The neonatal ICU (NICU) decided to revert back to paper because they felt that the functionality of the current system being utilised at King's did not offer them exactly what they needed on a daily basis. The EPMA team are looking into developing the suggestions made by NICU, with a hope that they will attempt another go-live. Currently, King's has $92 \%$ of all inpatient locations live with EPMA. The biggest lesson to learn is that there is fear associated with EPMA in paediatric areas, perhaps because of the delicate nature of the patient cohort. However, a well-designed project and constant engagement really allow for a smooth transition from paper to electronic. It is staggering to think that EPMA is the now regarded as essential for normal daily functional status at King's.

\section{Conflict of interest statement}

None. 